AperTO - Archivio Istituzionale Open Access dell'Università di Torino

\title{
Antichi marginalia nei Florida di Apuleio
}

\section{This is the author's manuscript}

Original Citation:

Availability:

This version is available http://hdl.handle.net/2318/154321

since

Terms of use:

Open Access

Anyone can freely access the full text of works made available as "Open Access". Works made available under a Creative Commons license can be used according to the terms and conditions of said license. Use of all other works requires consent of the right holder (author or publisher) if not exempted from copyright protection by the applicable law. 
This is the author's final version of the contribution published as:

Giuseppina Magnaldi. Antichi marginalia nei Florida di Apuleio. RIVISTA DI FILOLOGIA E DI ISTRUZIONE CLASSICA. 142 (2) pp: 376-407.

When citing, please refer to the published version.

Link to this full text:

http://hdl.handle.net/2318/154321 


\section{ANTICHI MARGINALIA NEI FLORIDA DI APULEIO}

Abstract: A number of marginalia that subsequently crept into the text transmitted for Apuleius' Florida are singled out and a new constitutio of some loci vexati is suggested.

Keywords: Apuleius, Florida, marginalia, textual criticism.

1. La recente collazione autoptica svolta da F. Piccioni del codice Ambrosiano N 180 Sup. (A, sec. XIII' ${ }^{1}$ ), in vista dell'edizione oxoniense di Apologia e Florida di Apuleio, sembra confermare che il manoscritto, comunemente ritenuto il migliore rappresentante dei recenziori, dipenda anche per queste due opere dall'autorevolissimo Laurenziano $68.2\left(\mathrm{~F}\right.$, sec. XI) ${ }^{1}$, come già avevano argomentato per le Metamorfosi C. Giarratano (Augustae Taurinorum 1929, $1960^{2}$ a cura di P. Frassinetti), D. S. Robertson (Paris 1940-1945, rist. 19651971) e M. Zimmerman (Oxonii 2012). Sia nei fogli contenenti il 'romanzo' sia in quelli che conservano le opere oratorie, A è una copia di F che, sia pure mediata dal perduto a ('padre' della I classe dei recenziori, compreso il codice utilizzato da J. A. De Buxis per l'editio princeps del 1469), risulta spesso più fedele al modello di quanto non lo sia $\varphi=$ Laurenziano 29.2, direttamente esemplato da F a Montecassino intorno al 1200. Tramite il controllo incrociato di A e di $\varphi$ si riesce a ricostruire la facies di $\mathrm{F}$ in un buon numero di luoghi in cui oggi questo prezioso manoscritto non è più leggibile per schiarimento dell'inchiostro o rasura o sovrapposizione di mani o guasti materiali di varia natura. In tal senso, il contributo di A è imprescindibile per la constitutio textus di Metamorfosi, Apologia e Florida, come giustamente affermano Zimmerman e Piccioni, ma la speranza di trovare nel codice lezioni indipendenti atte a sanare le gravi corruttele pre-

\footnotetext{
${ }^{1}$ Così, per l'Apologia, Piccioni 2011, come già Magnaldi 2000, 2004²b (sulla base della collazione in microfilm e fotografia di A e di F). Piccioni mi comunica che i dati di collazione dei Florida sono in corso di stampa, ma che la conclusione stemmatica è analoga.
}

RFIC, 142, 2014, 376-407 
senti nelle tre opere si riduce all'eventuale sporadica migrazione in a di qualche variante extrastemmatica ${ }^{2}$.

Sembrerebbe pertanto inevitabile rassegnarsi nella maggior parte dei loci vexati all'alea rischiosa dell'emendatio puramente congetturale, se per alcuni di essi una possibile terza via fra lezione tràdita e congettura non fosse stata indicata da R. Helm nella Praefatio anteposta all'edizione teubneriana dei Florida del 1910. Qui l'editore dedica grande attenzione ai vetusti marginalia che il copista di F ha tanto fedelmente quanto meccanicamente tramandato all'interno del testo. Il primo gruppo è costituito dalle glosse intruse in linea nelle vicinanze delle parole glossate (de interpretamentis additis); il secondo dalle correzioni compresenti con i rispettivi errori (de lectionibus rectis et falsis in unum coactis) ${ }^{3}$. Il testo e l'apparato allestiti dall'editore provano con evidenza che l'espunzione delle glosse e la messa in atto delle correzioni - in altre parole, il ripristino della lezione più antica - consentono di sanare non poche corruttele di Metamorfosi, Apologia e Florida.

In anni recenti, questa linea di ricerca sui marginalia tràditi in modo acritico da F è stata da me proseguita per le Metamorfosi $^{4}$, con acquisizioni testuali positivamente accolte dalla comunità scientifica, come mostra da ultimo l'edizione oxoniense di Zimmerman. Sto ora esaminando dallo stesso punto di vista i Florida, allo scopo di rintracciare anche in quest'opera, molto meno studiata delle Metamorfosi, eventuali duplices lectiones misconosciute. Presenterò qui le prime individuazioni, alcune delle quali sfociano in nuove proposte di constitutio textus, mentre altre rafforzano interventi testuali già eseguiti nei secoli scorsi ma sottovalutati dagli editori moderni.

Nell'apparato che allestirò per i singoli luoghi (circoscrivendolo alle lezioni di volta in volta discusse) farò costante riferimento alle edizioni critiche («edd.») di R. Helm (Lipsiae 1910, rist. 1959 cum addendis) e di P. Vallette (Paris 1924, 19602) $)^{5}$. Ad esse si aggiunge-

2 Sull'intenso scambio di varianti fra i testimoni di Apuleio a Montecassino cfr. Pecere 1987, $2003^{2}$.

${ }^{3}$ Helm 1910, 1959², LIII-LV.

${ }^{4}$ Magnaldi 2000, 2004²b e 2008.

5 Eviterò tuttavia la generica sigla $v=$ vulgata (spesso utilizzata dai due editori), citando invece nominativamente gli autori dei singoli emendamenti. In tutte le citazioni desunte da traduzioni e da edizioni antiche e moderne (adnotationes e apparati) è sottintesa l'indicazione «ad loc.». Per 
ranno, ove necessario, la traduzione con testo critico di G. Augello (Torino 1984), le edizioni commentate di V. Hunink (Amsterdam 2001) e di B. T. Lee (Berlin-New York 2005) e la traduzione di J. L. Hilton (Oxford 2001).

2. Incominciamo con le glosse, la cui presenza nel testo tràdito dei Florida sarebbe massiccia secondo alcuni studiosi, scarsa secondo altri. Tale divergenza di giudizio si spiega con la difficoltà di pronunciarsi sull'autenticità o meno di particolari nessi ridondanti, essendo la ridondanza abituale in tutte le opere di Apuleio, e largamente praticata in questi frammenti oratori che dello stile 'parlato' propongono con grande efficacia i moduli espressivi. Di qui la frequenza sia delle ripetizioni e quasi-ripetizioni (destinate a riprendere le fila del discorso o improntate all'enfasi o riecheggianti le movenze ingenue del racconto aneddotico) sia delle parafrasi (volte a chiarire concetti complessi ripresentandoli in forma semplificata) sia dei cumuli sinonimici (che mirano di volta in volta a specificare il significato contestuale di una parola, a fissare l'attenzione del pubblico su oggetti degni di rilievo, a produrre effetti musicali).

Ecco qualche esempio utile a illustrare l'atteggiamento critico degli studiosi apuleiani nei confronti di espressioni ridondanti particolarmente significative. Mentre nessun sospetto è mai stato sollevato sulla frequente ripresa strutturale di termini posti a notevole distanza l'uno dall'altro (quali Protagora... eum Protagoran in 18, 19-20 Protagora, qui sophista fuit longe multiscius... eum Protagoran aiunt, o navem... eam navem in 23, 1-2 sicuti navem bonam fabre factam... eam navem si aut gubernator non agat aut tempestas agat), qualche dubbio hanno destato sia l'iterazione 'narrativa' Creophyli...qui Creophylus di 15, 21 itemque Leodamantem Creophyli discipulum, qui Creophylus memoratur poetae Homeri hospes et aemulator canendi fuisse, sia l'iterazione 'enfatica' in illa curia... in qua curia di 16, 44 qui me in illa curia honestissimis adclamationibus decoravere, in qua curia vel nominari tantummodo summus honor est. In entrambi i casi G. Krüger (Berolini 1865) ha espunto come glosse le parole ripetute in seconda sede (rispettivamente Creophylus e curia $^{2}$ ), ma gli editori moderni hanno giustamente respinto le sue proposte, citandole

l'inquadramento contestuale di tutti i passi qui discussi, importante punto di riferimento è Harrison 2000, 89-130. 
in apparato come congetture dal semplice valore diagnostico (così Helm) o sottacendole (così Vallette).

Maggiori sospetti si sono addensati su alcune espressioni sinonimiche, sebbene Apuleio si serva spesso di sinonimi per i fini più diversi: basti citare 21, 6 fruticem, quem verberando equo gestant, eam virgam in laevam manum transferunt, in cui il frutex viene ripreso e variato in eam virgam per visualizzare con più nettezza quella frascascudiscio e per mimarne il suono tramite gli echi allitteranti che il sinonimo produce. Ciononostante, più di uno studioso ha manifestato dubbi su pedum tegumenta crepidas di 9, 21, che Helm e Vallette stampano così: Etiam pedum tegumenta crepidas sibimet compegerat; etiam anulum in laeva aureum faberrimo signaculo quem ostentabat, ipse eius anuli et orbiculum circulaverat et palam clauserat et gemmam insculpserat. In questo passo sulla sapienza artigiana del sofista Ippia, che in nome dell' $\alpha$ ứó $\chi \varepsilon 1 \alpha$ si fabbricava da solo indumenti, calzari e ornamenti, F. Oudendorp (Lugduni Batavorum 1823, a cura di J. Bosscha) propone in apparato di mutare etiam pedum tegumenta in et in pedum tegumenta, Krüger espunge come glossa crepidas, E. Rohde ritocca l'accusativo tegumenta nel dativo tegumento ${ }^{6}$. Ma l'astratto tegumenta («protezioni») è apposizione del concreto crepidas («sandali»), e la somma delle due parole, grazie alla trama onomatopeica che produce, appare del tutto congruente con la minuzia fonico-descrittiva di 9, 17 et indumenta quibus indutus et calciamenta quibus erat inductus. Analogo virtuosismo linguistico spiega bene tunicam interulam («camicia») in 9, 18 Habebat indutui ad corpus tunicam interulam tenuissimo textu, triplici licio, purpura duplici. Non si consentirà perciò con L. Nougaret, che espunge tunicam per simmetria con 9, 19 habebat cinctui balteum e 9, 20 habebat amictui pallium candidum e per analogia con met. 8, 9, 2 (dove interula compare da solo come aggettivo sostantivato) ${ }^{7}$.

Uno scopo diverso sembra aver perseguito Apuleio con due pericopi sinonimiche introdotte rispettivamente da scilicet e da id est, forse poco eleganti ma utili a spiegare agli ascoltatori in modo semplificato concetti non immediatamente perspicui. La prima si trova in 2, 1-2 At non itidem maior meus Socrates, qui, cum decorum adulescentem et diutule tacentem conspicatus foret, «ut te videam» inquit «aliquid

\footnotetext{
${ }^{6}$ Rohde $1885,110$.

7 Nougaret 1928, 42-46. Secondo Marangoni 2000, 22-23, la minuta descrizione di questa tunica riecheggia Verg. Aen. 5, 250-251 e 258-260.
} 
et loquere». Scilicet Socrates tacentem hominem non videbat; etenim arbitrabatur homines non oculorum, sed mentis acie et animi obtutu considerandos. Qui Hunink confuta efficacemente l'atetesi di scilicet tacentem hominem non videbat, eseguita da Nougaret in quanto «cette explication... est introduite par scilicet... est toute entière tirée du contexte... fait double emploi avec la suite du passage etenim arbitrabatur» ${ }^{8}$. Nessun commento dedicano invece i due studiosi a un'altra proposta di espunzione autorevolmente avanzata da J. Floridus (Parisiis 1688) per la chiusa di 16, 25 An non properandum mihi erat, ut pro eo honore vobis multas gratias dicerem pro quo nullas preces dixeram? Non quin magnitudo Carthaginis mereatur etiam $<a>$ philosopho precem pro honore, sed ut integrum et intemeratum esset vestrum beneficium si nihil ex gratia eius petitio mea defregisset, id est, ut usque quaque esset gratuitum. Apuleio sta qui ringraziando i Cartaginesi per aver decretato la costruzione di una statua in suo onore senza che egli ne avesse fatto esplicita richiesta. L'editore ad usum Delphini esprime in nota forti dubbi sull'appendice id est... gratuitum, un po' pedestre dopo la sofisticata solennità del ragionamento precedente: «Videntur haec verba a manu Glossatoris textui postea inserta». Sbaglia Vallette a non citare in apparato (a differenza di Helm) la congettura di Floridus, che non merita forse di essere accolta ma serve tuttavia a riflettere su Apuleio glossatore di se stesso.

Sempre in flor. 16, Floridus aveva interpretato come glossa, sulla scia di molti predecessori (G. Scioppius, Lugduni Batavorum 1594; J. Wowerius, Hamburgi 1606; G. Elmenhorstius, Francofurti a. M. 1621; P. Scriverius, Amstelodami 1624), un'altra proposizione collocata nella parte iniziale del discorso, là dove Apuleio, per giustificare il ritardo con cui si è presentato ai Cartaginesi, introduce il confronto fra un incidente occorsogli in palestra e quello, altrettanto imprevedibile ma molto più grave, capitato secoli prima al comico Filemone. La constitutio textus di Helm e di Vallette è la seguente: 16, 1-5 ... volo causam vobis allegare cur aliquam multos dies a conspectu auditorii afuerim contulerimque me ad Persianas aquas, gratissima prorsus et sanis natabula et aegris medicabula - quippe ita institui omne vitae meae tempus vobis probare quibus me in perpetuum firmiter dedicavi: nihil tantum, nihil tantulum faciam quin

8 Nougaret 1928, 45; Hunink 2001, 63. Già Opeku 1974, 57 aveva obiettato a Nougaret che senza quella frase «the sequence of thought would, however, be less clear». 
eius vos et gnaros et iudices habeam - quid igitur de repentino ab hoc splendidissimo conspectu vestro distulerim. Exemplum eius rei paulo secus simillimum memorabo, quam improvisa pericula hominibus subito oboriantur, de Philemone comico. De ingenio eius qui satis nostis, de interitu paucis cognoscite. L'interpunzione, con l'inciso quippe... habeam fra lineette e con il punto fermo dopo distulerim, è di Helm, che suggerisce in apparato di interpretare quid... distulerim quale ripresa della lontana interrogativa cur... afuerim (oppure, meno plausibilmente, di sottintendere vultis scire $)^{9}$. Prima di Helm, gli editori interpungevano dopo habeam, riferendo Quid... distulerim a eius rei e cercando di sbrogliare in vari modi l'intrico sintattico risultante. De Buxis e B. Philomathes (curatore della seconda edizione Giuntina del 1522) segnano un punto fermo davanti a De Philemone comico, poi modificato in virgola (così G. F. Hildebrand, Lipsiae 1842); Oudendorp traspone la proposizione quam... oboriantur dopo eius rei e Krüger davanti a eius rei; Floridus consente in nota con l'espunzione della frase ad opera di Scioppius e della maggior parte degli editori secenteschi: «Ista abundant, videnturque a manu Glossatoris esse, et irrepsisse in textum». È arduo, tuttavia, attribuire a un glossatore una frase tanto linguisticamente elaborata, anche se sui Florida hanno senza dubbio 'lavorato' studiosi competenti, a partire dallo stesso excerptor che ha allestito la raccolta. Quella frase sarà piuttosto un inciso esplicativo dell'autore, una sorta di titolo sotto cui gli ascoltatori dovevano incasellare l'esemplare storia di Filemone.

3. Mentre nei passi fin qui citati l'usus apuleiano consente di difendere parole e frasi espunte come spurie dall'uno o dall'altro vir doctus, nel luogo ora in discussione (dedicato alla celeberrima sfida musicale fra Marsia e Apollo) un'antica glossa sembra essersi effettivamente insediata in linea, cacciando e sostituendo la vera lectio. È questo il persuasivo giudizio espresso da C. Brakman e accolto in apparato da Vallette, che però continua a stampare nel testo la chiosa tibicen, come tutti gli editori antecedenti. Per visualizzare l'accaduto, si dovrà secludere tibicen e porre subito di seguito il segno di lacuna. Nello stesso passo un'altra glossa, già espunta da Krüger (come ricorda Helm in apparato), è stata a torto trascurata da Vallette e dai successivi interpreti apuleiani. Le due individuazioni sfociano nel testo seguente:

\footnotetext{
${ }^{9}$ Condivide la prima interpretazione Toschi 2000, 57.
} 
3, 8 Sed Marsyas, quod stultitiae maximum specimen, non intellegens se deridiculo haberi, priusquam tibias occiperet inflare, prius de se et Apolline quaedam deliramenta barbare effutivit, laudans sese, quod erat et coma relicinus et barba squalidus et pectore hirsutus et arte [tibicen] $<_{* * *}>$ et fortuna egenus: 9 contra Apollinem - ridiculum dictu - adversis virtutibus culpabat, quod [Apollo] esset et coma intonsus et genis gratus et corpore glabellus et arte multiscius et fortuna opulentus.

tibicen ut glossema seclusi lacunamque indicavi (cf. Vallette in app.: «certe videtur tibicen in textum irrepsisse auctorisque manum expulisse»): unimodus Brakman (coll. Plat. 227 unimodam... virtutem), uniformis vel unicanus Hunink (coll. met. 11, 5, 1 deorum dearumque facies uniformis et flor. 13, 3 philosophi ratio et oratio... omnicana) // Apollo del. Krüger, servant edd.

Sulla scorta di Vallette, un'accurata analisi della prima parte del passo è stata condotta da Hunink, secondo il quale, al posto di una parola «surprisingly weak» come tibicen, ci si aspetterebbe «a more remarkable and sonorous epithet», in conformità con squalidus, hirsutus ed egenus e in antitesi con multiscius. Che tibicen e multiscius possano opporsi l'uno all'altro, come voleva L. C. Purser, in quanto «flautista autentico» (soltanto flautista) e «dilettante» (praticante molteplici arti), lo aveva già escluso F. Opeku: «this misses the point that Marsyas is here represented as reversing the true values, and praising himself for virtues that are none». Proseguendo dunque nella direzione di ricerca indicata da Brakman con unimodus, Hunink propone uniformis oppure unicanus («producing music in one tone»), non attestato nella lingua latina ma coniato sul neologismo apuleiano di flor. 13, 3 omnicana ${ }^{10}$. Tuttavia, al di là della irrecuperabile lezione genuina scalzata da tibicen, occorre indagare le ragioni che sottostanno alla glossa, ovvero chiedersi perché mai sia stato ripetuto a margine l'epiteto di «flautista», attribuito dall'autore a Marsia in 3,1 e 3,13. Aiuta a rispondere la ripetizione poco successiva di Apollo, espunto per la sua fastidiosa superfluità dopo

${ }^{10}$ Hunink 2001, 75 e n. 1; Opeku 1974, 84-85; Purser 1907, 401. Le riflessioni dei tre studiosi assumono quale punto di partenza l'analisi di Brakman 1908, 30-31: «Porro unusquisque intellegit, quam male tibicen et multiscius, quam optime cetera adiectiva inter se opponantur. Itaque mireris, cur peritissimus verborum artifex non dederit unimodus ac profecto fieri potest, ut tibicen suprascriptum vel in margine adscriptum in textum irrepserit ipsiusque auctoris manum expulerit». 
contra Apollinem prima da Krüger e poi da J. van der Vliet (Lipsiae 1900). Helm e Opeku difendono la ripetizione, che mirerebbe per il primo alla massima chiarezza, in sintonia col tono colloquiale del passo, e per il secondo alla «contrastive emphasis» ${ }^{11}$. Ma l'analisi congiunta di tibicen e di Apollo suggerisce piuttosto che entrambi i termini siano vetusti lemmi, vergati a margine allo scopo di attirare lo sguardo sulla descrizione contrapposta dei due contendenti, tanto più efficace per il paradossale rovesciamento di valori che effettua Marsia.

4. Oltre ai lemmi di flor. 3, 8-9, sono verisimilmente penetrate nel testo tràdito dei Florida altre due glosse, che rientrano tra le specie più diffuse: l'esplicitazione di un termine sottinteso (commemorant) e la spiegazione sinonimica di un termine raro (operibus). Con l'espunzione di commemorant ad opera di J. Tollius (citato da Oudendorp $)^{12}$ il primo passo si configura nel modo seguente:

6, 9 Nec quicquam aeque penes illos laudo, quam quod torporem animi et otium oderunt. 10 Igitur ubi mensa posita, priusquam edulia adponantur, omnes adolescentes ex diversis locis et officiis ad dapem conveniunt; magistri perrogant quod factum a lucis ortu ad illud diei bonum fecerint. 11 Hic alius se commemorat inter duos arbitrum delectum, sanata simultate, reconciliata gratia, purgata suspicione amicos ex infensis reddidisse; 12 itidem alius sese parentibus quaepiam imperantibus oboedisse, et alius aliquid meditatione sua repperisse vel alterius demonstratione didicisse; denique ceteri [commemorant]. Qui nihil habet adferre cur prandeat, inpransus ad opus foras extruditur.

denique ceteri [commemorant] Tollius: post commemorant lacunam statuit Krüger, [denique ceteri commemorant] Müller (Brakman), denique $<$ cetera > ceteri commemorant van der Vliet, <sua > denique ceteri commemorant Leo, <alia > denique ceteri commemorant Helm, lacunam ante denique statuit Vallette, denique [ceteri] commemorant (commate ante denique deleto) Hunink in adnotatione.

${ }^{11}$ Helm 1904, 516 (con lui consente Brakman 1908, 30, n. 1); Opeku 1974, 85. Tacciono sul problema Vallette, Hunink e Lee.

12 Di Jacobus Tollius (1633-1696), editore di Ausonio, Oudendorp afferma (t. II 286) di riportare alcune note vergate a margine di una copia dell'edizione apuleiana di Scriverius. Essa si identifica probabilmente con il Liber Annotatus Tollii, oggi in possesso della Bibliotheek der Rijksuniversiteit di Leida (che l'acquistò a un'asta nel 2004). 
In questo passo, dedicato al mirabile tirocinio cui in India i ginnosofisti sottopongono i loro allievi, Tollius (approvato in nota da Oudendorp) ha espunto commemorant, che sembra la puntigliosa esplicitazione, ad opera di un glossatore, del verbo lasciato sottinteso da Apuleio al termine di un lungo elenco di soggetti e di azioni saldamente retto dal commemorat iniziale. Senza commemorant, vanamente difeso da H. Armini nel senso assoluto di «verba faciunt» 13 , la chiusa denique ceteri è più agile $\mathrm{e}$ meno pedante di quella che risulta dall'integrazione di un complemento oggetto quale cetera o sua o alia, ad opera rispettivamente di van der Vliet, F. Leo (seguito da Augello) e Helm. Un uso non troppo dissimile di cetera con verbo inespresso (e indeterminato: il contesto suggerisce genericamente il significato, non il significante) si trova in met. 4, 8, 5 Clamore ludunt, strepitu cantilant, conviciis iocantur, ac iam cetera semiferis Lapithis evantibus Centaurisque similia (è sottinteso faciunt o un verbo sinonimico) e in apol. 22, 5 Iam cetera tam mirifica quae, si tu legisses, magis mihi peram quam nuptias Pudentillae invidisses (è sottintesa un'espressione del tipo scripsit Crates).

Nel passo in discussione, dunque, dopo alius, poi itidem alius, poi et alius, che ricordano ad uno ad uno le proprie buone azioni, Apuleio ha chiuso l'elenco con l'espressione ellittica denique ceteri, e lo ha fatto al fine di sottolineare che l'interrogatorio dei ginnosofisti riguardava «tutti gli altri» giovani, anche quelli sul cui pubblico esame di coscienza egli evita di dilungarsi. Queste due parole vanno pertanto conservate, contrariamente a quanto pensano da un lato $\mathrm{H}$. Müller e Brakman, che espungono come «emblema» denique ceteri commemorant ${ }^{14}$, e dall'altro Hunink che, pur ponendo con Vallette il segno di lacuna davanti a denique (così anche Lee), suggerisce in nota di secludere ceteri (e di eliminare la virgola dopo didicisse) ${ }^{15}$.

13 Armini 1928, 330-331. Ma in Apuleio commemoro è sempre accompagnato da un complemento oggetto o da un'infinitiva.

14 Müller 1867, 648: «denique ceteri commemorant ist wohl Zusatz eines Grammatikers»; Brakman 1908, 32: «Post alius iteratum in tribus periodi membris, quae describunt bona facta adulescentium a magistris interrogatorum, mire languent verba: denique ceteri commemorant, quae nemo desiderat. Immo illis verbis, quae emblema olent, omittendis sententiam Apuleio dignam reddis».

${ }^{15}$ Hunink 2001, 93, n. 1: «Alternatively, one might consider not inserting a word, but deleting ceteri and omitting the comma after didicisse: the verb commemorant would thus be governed by the preceding subjects itidem alius... et alius». 
Del resto, mentre l'esplicitazione di un verbo sottinteso come commemorant è un tipo di glossa che si riscontra con grande frequenza in tutti i codici, non si capisce perché mai qualcuno avrebbe sentito l'esigenza di postillare il testo aggiungendo ceteri o denique ceteri commemorant ${ }^{16}$.

5. Veniamo ora alla glossa operibus, volta a spiegare il raro opifici$i s$. La sua espunzione ad opera di Wowerius sfocia nel testo seguente:

9, 13 Patior et ipse in meis studiis aliquam pro meo captu similitudinem; nam quodcumque ad vos protuli, excerptum ilico et lectum est, nec revocare illud nec autem mutare nec emendare mihi inde quicquam licet. 14 Quo maior religio dicendi habenda est, et quidem non in uno genere studiorum. Plura enim mihi exstant in Camenis quam Hippiae in opificiis [operibus]. Quid istud sit, si animo attendatis, diligentius et accuratius disputabo. 15 Et Hippias e numero sophistarum est, artium multitudine prior omnibus, eloquentia nulli secundus (...). 17 Omnia secum quae habebat, nihil eorum emerat, sed suis sibi manibus confecerat, et indumenta quibus indutus, et calciamenta quibus erat inductus, et gestamina quibus erat conspicatus.

excerptum $\mathrm{F} \varphi$ : exceptum Philomathes (edd.) ${ }^{17}$ // in opificiis [operibus] Wowerius: in [opificiis] operibus Vulcanius, in opificiis opera (vel in opificis operibus) Scioppius (edd.), in opificii[s] operibus Oudendorp in app. («posset et scribi opificii, sive manuum»), in opificiis [opera] Augello.

I dubbi sulla lezione tràdita in opificiis operibus (accolta dall'editio princeps) attraversano i secoli, e fanno sentire i loro effetti anche sui più autorevoli lessici moderni. Il TLL recepisce dubitativamente opificiis sotto il lemma opificius (accompagnato da punto interrogativo: «si genuinum, ab opifex»), e lo interpreta come aggettivo: «i. q. ad opificem pertinens». A un aggettivo, ma dal nominativo opificus,

16 Secondo Opeku 1974, 121 «It is possible that more of the text has been lost here than has been generally suspected, and that what we have is an early attempt to fall a larger gap».

17 Come suggeriscono sia Hunink 2001, 109-110 (e poi Hunink 2001b, 321-324) sia Lee 2005, 98, va accolta la lezione tràdita excerptum = «estratto» contro l'emendamento di Philomathes exceptum = «annotato», stampato da quasi tutti gli editori successivi e recentemente difeso da Vössing 2008, 395-397. Ma sulla questione occorrerà tornare in modo più approfondito. 
già pensava Scioppius con la congettura in opific[i]is operibus (così stampa Hildebrand, per analogia con lanificus). Maggiore fortuna riscuote l'altra congettura proposta da Scioppius, in opificiis opera, che entra stabilmente nel testo da inizio Seicento fino alle edizioni di Helm e di Vallette e ai commenti di Hunink e di Lee. La accoglie anche l'OLD, interpretando opificium quale sostantivo: «The performance of constructive work».

Credo anch'io che Apuleio abbia qui usato il sostantivo opificium $-i$, attestato prima di lui in Varrone rust. 3, 16, 20 nisi opifici eas (sc. apes) urget tempus (il plurale opificia si trova in autori tardi, «but seems not impossible», come commenta Hunink) ${ }^{18}$. Proprio perché si tratta di un vocabolo raro, l'autore invita il suo pubblico a ricavarne il significato da ciò che dirà poco dopo (Quid istud sit, si animo attendatis, diligentius et accuratius disputabo), ovvero dalle parole omnia secum quae habebat... suis sibi manibus confecerat, con ricchissima enumerazione dei manufatti più diversi, dagli indumenti alle calzature ai gioielli agli accessori da bagno. In considerazione di tutto ciò, un lettore avrà glossato opificiis con operibus, opportunamente espunto da Wowerius (già B. Vulcanius, Lugduni Batavorum 1594, aveva semplificato il cumulo sinonimico in opificiis operibus, ma alla rovescia, espungendo il difficile opificiis e conservando il più banale operibus $)^{19}$.

Il testo che risulta dall'atetesi è migliore di quello stampato da Helm e da Vallette (Plura enim mihi exstant in Camenis quam Hippiae in opificiis opera: «car mes travaux dans le domaine des Muses sont plus nombreux que ceux d'Hippias dans les arts manuels»), perché permette di sottintendere genera, e in particolare, per Apuleio, genera studiorum, «composizioni appartenenti a più generi letterari», in piena conformità sia con le parole precedenti non in uno genere studiorum (è chiaro qui che studium vale «opera letteraria»: si veda anche poco prima in meis studiis) sia con il seguito delle argomentazioni apuleiane: cfr. 9, 27-29, dove Apuleio oppone ai manufatti di Ippia i suoi poemata omnigenus apta virgae, lyrae, socco, coturno, item satiras ac griphos, item historias varias rerum nec non orationes laudatas disertis nec non dialogos laudatos philosophis atque

18 Hunink 2001, 110 e n. 2. Sorvola sulla questione Lee.

${ }^{19}$ Così giustamente osserva Oudendorp: «Vulcanius delendum censebat opificiis. At si alterutra vox glossema est, videtur esse potius operibus, quod censebat Wowerius». 
haec et alia eiusdem modi tam graece quam latine, gemino voto, pari studio, simili stilo 20 .

6. Più incerta è la presenza di una glossa nel luogo seguente, uno tra i più problematici dei Florida, ma vale comunque la pena di proporre il testo che risulterebbe dall'individuazione:

2, 8 Aquila enimvero cum se nubium tenus altissime sublimavit evecta alis totum istud spatium qua pluitur et ninguitur, ultra quod cacumen nec fulmini nec fulguri locus est, in ipso, ut ita dixerim, solo aetheris et fastigio hiemis - 9 cum igitur eo sese aquila extulit, nutu clementi laevorsum vel dextrorsum tanta mole corporis labitur, velificatas alas quo libuit advertens modico caudae gubernaculo; 10 inde cuncta despiciens [ibidem], pinnarum eminens indefessa remigia ac paulisper cunctabundo volatu paene [eodem loco] <ibidem> pendula circumtuetur et quaerit quorsus potissimum in praedam superne sese ruat fulminis vicem eqs.

ante inde dist. Oudendorp // ibidem transposui, verbis eodem loco ut glossa seclusis: inhibens Heinsius, innitensque Oudendorp, itidem dub. Krüger // eminens Helm (Vallette, coll. met. 2, 21, 2 infimis conclusis digitis ceteros eminens): eminus $\mathrm{F} \varphi$, del. Krüger, post despiciens transp. van der Vliet, imminuens Goldbacher, deminuens Purser, alii alia // indefessa remigia $\mathrm{F} \varphi$ : indefessa remigio Scaliger, indefesso remigio Floridus.

Un serio problema con cui si sono confrontati editori e interpreti è l'interpunzione dopo cum igitur, del tutto assente nell'editio princeps. Nella Giuntina del 1522 Philomathes, sempre vigile sulla distinctio, non interpunge davanti a inde, ma davanti a ibidem, seguito dalla maggior parte degli editori successivi fino a Oudendorp. Questi segna invece due virgole, una prima di ciascun avverbio, e così fanno anche Hildebrand e Hunink ${ }^{21}$, mentre Helm e Vallette (seguiti da Augello e Lee) eliminano la virgola davanti a ibidem. Tali oscillazioni lasciano trasparire l'imbarazzo determinato dal cumulo inde (moto da luogo) + ibidem (stato in luogo), che appare difficilmente accettabile an-

${ }^{20}$ Non coglie il riferimento di plura a genera Augello 1984, 422, che seclude «per ragioni di miglior senso» il vulgato opera (sarebbe stato metodologicamente più corretto secludere il tràdito operibus) ma poi traduce, genericamente, «Giacché le mie attività nel campo delle muse sono più numerose di quelle di Ippia nel campo dei lavori manuali».

${ }^{21}$ Quest'ultimo aggiunge però un punto e virgola dopo remigia, perché ac paulisper sarebbe «the beginning of a second main sentence»: Hunink 2001, 67. 
che dopo l'acuto e largamente condiviso emendamento ad opera di Helm di eminus in eminens = porrigens ( $«$ thrusting out his wings like untiring oars», secondo l'efficace resa di Hilton). Vallette e Augello evitano di tradurre i due avverbi (limitandosi rispettivamente a «embrassant d'un coup d'oeil l'espace qu'il domine» e «osservando tutto lo spazio che domina»), mentre Hilton traduce soltanto inde: «looking down from there on everything».

Prima di loro, Krüger proponeva di mutare in itidem l'avverbio ibidem, mentre A. J. Kronenberg tentava di difenderlo forzandone il significato: ibidem sarebbe un avverbio di tempo equivalente a simul e dunque inde cuncta despiciens ibidem varrebbe inde cuncta simul despiciens $^{22}$. Diversa era stata l'interpretazione di Oudendorp, che sulla scia di Floridus aveva collegato ibidem con il tràdito eminus: «illinc deorsum intuens omnia, ibidem ex alto» ${ }^{23}$. Forti dubbi, tuttavia, venivano da lui avanzati in nota: «Nec etiam video, quid hic faciat vox ibidem. Ea omnino mihi suspecta est et mendae sedes». Seguono la citazione dell'emendamento di N. Heinsius et inhibens (che sarà accolto da van der Vliet) e poi la proposta sua propria, sulla base di Ov. met. 7, 401: «Quare vide num scribi possit innitensque pinnarum eminus indefessa remigio. Hac in re certe 'inniti' non infrequens est».

Ora, se si osserva che ibidem per un verso appare fuori posto là dove lo tramandano i codici e per l'altro esprime con più eleganza lo stesso significato di eodem loco, nasce il sospetto che eodem loco sia una glossa, confluita nel testo al posto della parola glossata ibidem. Chi ha operato la sostituzione avrebbe dislocato a margine quale variante ibidem, che in un successivo stadio di tradizione sarebbe ritornato in linea un po' prima del punto di partenza. Tale spiegazione genetica della corruttela può sembrare audace, ma nel testo risultante inde cuncta despiciens, pinnarum eminens indefessa remigia ac paulisper cunctabundo volatu paene ibidem pendula circumtuetur et

22 Kronenberg 1908, 312, con citazione di apol. 80 ut absurde facit qui tacere se dicit, quod ibidem dicendo tacere sese non tacet. Secondo Opeku $1974,66-67$, «ibidem (if retained) = 'at the same spot' or 'at the same moment'» potrebbe collegarsi «either with what follows or with what precedes».

${ }^{23}$ Così anche, recentemente, Martos 2011, 104-105, che difende eminus ma modifica con J. J. Scaliger (Lugduni Batavorum 1600) remigia in remigio, traducendo così: «looking down on everything from that point; there, so far off, tireless in rowing its wings». 
quaerit si elimina il problematico cumulo inde... ibidem e contemporaneamente si ottiene con paene ibidem pendula la riproduzione onomatopeica del soffice battito d'ali dell'aquila «quasi sospesa e oscillante in un unico punto» ${ }^{24}$. Lo specifico significato contestuale di ibidem, vocabolo di per se stesso né raro né difficile, avrebbe indotto un antico lettore a glossarlo con eodem loco.

7. Che i margini di antichi manoscritti dei Florida contenessero, oltre alle glosse, anche alcune variae lectiones, successivamente inglobate nel testo, è suggerito dai due luoghi ora in discussione. In entrambi van der Vliet ha espunto una parola fastidiosamente ripetuta, senza però trovare il consenso degli editori successivi. L'interpretazione di quelle ripetizioni, o quasi-ripetizioni, quali antiche varianti marginali può servire a rafforzare la sua constitutio textus. Ecco il primo luogo:

16, 33 Sed quaeram sedulo et conitar, 'dum memor ipse mei, dum spiritus hos regit artus'. Nam nunc inpraesentiarum - neque enim diffitebor - laetitia facundiae obstrepit et cogitatio voluptate impeditur; ac mens occupata delectatione mavult [inpraesentiarum] gaudere quam praedicare.

nunc del. Colvius (at cf. 16, 29 sed nunc in praesentiarum; 18, 12 tum etiam nunc inpraesentiarum) // inpraesentiarum ${ }^{2}$ secl. van der Vliet, servant edd.

Nel ringraziare i Cartaginesi per la statua a lui decretata, Apuleio si impegna a scrivere un libro in onore del magistrato Emiliano Strabone. Cercherà in futuro con tutte le sue forze (e qui cita Virgilio, Aen. 4, 336) di trovare le parole giuste; ora infatti glielo impedisce la troppa gioia. L'atetesi di inpraesentiarum ${ }^{2}$ ad opera di van der Vliet viene ricordata in apparato da Helm ma non da Vallette, che l'avrà evidentemente giudicata irrilevante. Del resto, la ripetizione era passata indenne attraverso tutte le edizioni anteriori al 1900, anche se P. Colvius (Lugduni Batavorum 1588) aveva sentito l'esigenza di 'perfezionarla' condannando nunc (ma nunc in praesentiarum ricorre in altri due luoghi dei Florida). Hunink, pur non registrando neppure lui la proposta di van der Vliet, ne tiene tacitamente conto nella sua difesa di inpraesentiarum ${ }^{2}$ : «The adverb... is used twice, after

${ }^{24}$ Analoga attenzione alla trama fonica si riscontra in flor. 6, 4 isdem Indis ibidem sitis. 
it has already occurred in 16,29 . The repetition may be intended to illustrate the speaker's difficulty in finding the right words, as he is overwhelmed by joy» 25 .

Tale giustificazione psicologica mi pare poco convincente. Il superfluo inpraesentiarum ${ }^{2}$ ha tutta l'apparenza di una parola spuria: non tanto la casuale dittografia commessa da un copista che torna con lo sguardo a nunc inpraesentiarum, quanto piuttosto una variante dimidiata di questa lezione. Il meccanismo genetico può essere così riassunto: un copista omette nunc (fra nam e inpraesentiarum l'avverbio è a rischio); un correttore lo integra in linea (nunc inpraesentiarum) ma disloca scrupolosamente a margine quale variante la decurtata lectio primitiva (inpraesentiarum), che rientrerà successivamente nel testo un po' dopo la lezione di riferimento ${ }^{26}$. Si potrebbe anche pensare, più semplicemente, che un antico lettore abbia trascritto a margine inpraesentiarum come parola degna di nota per la sua particolare formazione (in praesentia + rerum), ma questo avverbio ricorre già poco prima nello stesso discorso $(16,29)$, ed è là semmai che avrebbe dovuto attrarre l'attenzione.

8. Tra i vetusti marginalia intrusi nel testo tràdito dei Florida si dovrà verisimilmente annoverare anche confestim, espunto da van der Vliet nel luogo seguente:

19, 7 Postremo propinquis etiam hominibus invitis, quodne iam ipsi hereditatem habebant, an quod adhuc illi fidem non habebant, 8 aegre tamen Asclepiades impetravit brevem mortuo dilationem atque ita vispillonum manibus extortum velut ab inferis postliminio domum rettulit confestimque spiritum recreavit, [confestim] animam in corporis latibulis delitescentem quibusdam medicamentis provocavit.

confestim secl. van der Vliet (servant edd.): confestim $<q u e>$ Colvius, $<e t>$ confestim Krüger // animam<que $>$ van der Vliet.

In questa descrizione del salvifico intervento del medico Asclepiade, che richiama in vita un uomo da tutti ritenuto morto, la quasiripetizione confestimque... confestim è unanimemente accolta fino

\footnotetext{
${ }^{25}$ Hunink 2001, 166.

${ }^{26}$ Per esempi e riflessioni metodologiche su questo fenomeno, riscontrabile in molte tradizioni manoscritte, cfr. Magnaldi 2000, 142-153.
} 
a Colvius, che la perfeziona integrando dopo confestim ${ }^{2}$ l'enclitica que. Il suo intervento viene recepito dalla maggior parte degli editori successivi (compreso Oudendorp). Lo respingono invece Hildebrand e Helm, che lo cita tuttavia in apparato (pur attribuendolo genericamente alla vulgata), insieme con <et $>$ confestim di Krüger. Tacciono su entrambe le integrazioni Vallette, Augello, Lee e Hunink. Ma quest'ultimo sembra implicitamente tenerne conto nella sua difesa del testo tràdito: «the adverb was already used in 19.6 and is now repeted twice, probably to underscore Asclepiades' efforts» 27.

Queste argomentazioni, analoghe a quelle offerte per 16, 33 inpraesentiarum $^{2}$, sono troppo facili per essere persuasive, ma il luogo citato da Hunink offre interessanti spunti di riflessione: 19, 6 Confestim exclamavit vivere hominem: procul igitur faces abigerent, procul ignes amolirentur, rogum demolirentur, cenam feralem a tumulo ad mensam referrent. Nella seconda parte del passo si succedono proposizioni tutte in asindeto, le prime due introdotte entrambe da procul e la terza collegata alla precedente dalla 'rima' amolirentur... demolirentur (prima della chiusa cenam... referrent). Sulla scorta di questo luogo, in 19,8 si potrà semplicemente espungere confestim quale antica variante erronea del precedente confestimque (la decurtata lectio si spiega con l'estrema volatilità di -que, quasi sempre abbreviato), senza supplire dopo animam l'enclitica que, come invece fa van der Vliet. Confestimque si riferisce a entrambe le proposizioni spiritum recreavit e animam... provocavit, che non esigono alcuna congiunzione copulativa perché basta a congiungerle la forte somiglianza fonica dei due verbi in omeoteleuto recreavit e provocavit.

9. Anche nel passo ora in discussione una falsa lectio sembra essersi intrusa in linea un po' dopo la corrispondente recta. L'individuazione sfocia in una constitutio textus del tutto nuova.

9, 7 Quis enim vestrum mihi unum soloecismum ignoverit? Quis vel unam syllabam barbare pronuntiatam donaverit? Quis incondita et vitiosa verba temere quasi delirantibus oborientia permiserit blaterare? Quae tamen aliis facile et sane meritissimo ignoscitis. 8 Meum vero unumquodque dictum acriter examinatis, sedulo pensiculatis, ad limam et lineam certam redigitis, cum torno et coturno [verum] comparatis: tantum habet vilitas excusationis, dignitas difficultatis.

\footnotetext{
${ }^{27}$ Hunink 2001, 200.
} 
verum seclusi (ut variam lectionem ad antecedens vero attinentem): iterum editio princeps, ver $<b>$ um Goesius, $<a d>$ verum Vossius, $<$ in $>$ verum Hildebrand in adnot. (*verum* in textu), vero Leo (edd.).

Apuleio manifesta qui il timore di non riuscire a soddisfare le aspettative di un uditorio così esigente che non gli perdona nulla e misura ogni sua parola su lima e linea e la confronta con il tornio e il coturno tragico. Hunink e Lee si concentrano sulle virtuosistiche combinazioni ad limam et lineam e cum torno et coturno, e non commentano l'emendamento ad opera di Leo del tràdito verum in vero, accolto sia da Helm sia da Vallette. In precedenza gli editori recepivano dall'editio princeps la congettura iterum, che però Oudendorp confuta così in nota: «Pro iterum vero exhibent uterque Florentinus Pith. et Lips. verum: unde recte G. Vossium conjecisse reor ad verum. W. Goesius conjecit verbum» ${ }^{28}$. Anche Hildebrand, che stampa verum tra asterischi, rigetta iterum con la giustificazione seguente: «iterum quod edd. praebent per sententiam probari non posse videtur. Neque enim antea semel ipsa verba cum rei de qua disserebat qualitate vel quantitate comparaverant sed tum primum, cum illa audiverant... Ego malim in verum».

Pare tuttavia strano che Apuleio abbia inframmezzato all'ingegnosa espressione allitterante cum torno et coturno comparatis parole così insulse e fastidiosamente ripetute dopo vero ${ }^{1}$ quali $<a d>$ verum $\mathrm{o}$ $<$ in $>$ verum o vero. Non a caso quest'ultimo avverbio viene esplicitamente tradotto soltanto da Augello («ogni mia parola... la confrontate alla perfezione del tornio e del coturno tragico davvero» ${ }^{29}$, mentre

${ }^{28}$ Sbaglia Hildebrand ad attribuire a Vossius ad verbum.

29 Ossia, come spiega in nota, «volete un discorso ripulito e fine come per effetto del tornio e grave di sentenze da essere degno del coturno tragico» (Augello 1984, 459, n. 1). Le proposte <ad>verum o <in> verum o vero parrebbero a prima vista corroborate dall'aggettivo certam che accompagna lineam, ma linea, parola plurisemica, ha bisogno di venire in qualche modo qualificata per essere intesa quale strumento di misura (Opeku 1974, 149 interpreta «a line for measuring horizontally», con citazione di Cic. Quint. 3, 1, 2, Vitruv. 7, 3, 5, Plin. nat. 19, 147), mentre l'espressione cum torno et coturno basta a se stessa. È opportuno tuttavia riportare il suggerimento dell'anonimo referee cui devo un'approfondita lettura dell'articolo: «verum is clearly wrong, but it could be a corruption of a climatic adverb such as demum rather than an intrusion - some mark of climax here would be appropriate». 
Vallette sembra inglobarlo nel termine «perfection» («chacune de mes paroles... vous la mesurez à la perfection du tour et du cothurne tragique») e Hilton lo lascia inespresso («you... compare it with the products of the lathe or productions on the stage»). Con ogni probabilità, verum altro non è che variante erronea del precedente vero: un copista avrà dapprima scritto verum per vero, a causa dell'influsso di meum e unum(quodque); poi un correttore avrà emendato in linea, dislocando però la primitiva falsa lectio a margine; di qui essa sarà successivamente riconfluita nel testo, un po' dopo il punto di partenza. Oppure, più semplicemente, un lettore avrà annotato a margine, nelle vicinanze di vero, la variante verum, inglobata in linea in un successivo stadio di tradizione.

10. Dopo l'espunzione di glosse e di varianti erronee compresenti in linea con le lezioni di riferimento, mi occuperò ora di alcune particolari diplografie che sono forse indizio di altrettante 'integrazioni con parola-segnale'. Lavorando sulle Metamorfosi ho mostrato la frequenza nella tradizione apuleiana di questa particolare modalità suppletiva, che consisteva nel trascrivere a margine, insieme con la parola in un primo momento omessa, anche una o più parole precedenti o seguenti, già esattamente vergate ma ripetute allo scopo di indicare con la massima evidenza il luogo di lacuna. Basti qui citare alcuni esempi, accolti nel testo o approvati in apparato nella recente edizione oxoniense, prima di introdurre i casi analoghi presenti nei Florida.

Nel primo luogo la constitutio textus è già di Robertson:

met. 11, 1, 4 confestimque discussa pigra quiete <laetus et $>$ alacer exurgo meque protinus purificandi studio marino lavacro trado septiesque summerso fluctibus capite, quod eum numerum praecipue religionibus aptissimum divinus ille Pythagoras prodidit, [laetus et alacer] deam praepotentem lacrimoso vultu sic adprecabar (così stampa ora il testo Zimmerman; diplografia di alacer).

Nei tre esempi seguenti la constitutio textus è mia ${ }^{30}$ :

met. 1, 4, 2 Et <ante> tamen Athenis proximo [et ante] Poecilen porticum isto gemino obtutu circulatorem aspexi equestrem spatham praeacutam

${ }^{30}$ Magnaldi 2008, 431 e Magnaldi 2004²c, 64-68. 
mucrone infesto devorasse («fortasse recte» Zimmerman in apparato; diplografia di $e t$ );

met. 2, 25, 1-2 Sic desolatus ad cadaveris solacium, perfrictis oculis et obarmatis ad vigilias, animum meum permulcebam cantationibus, cum ecce crepusculum et nox <altior > provecta [et nox altior] et dein concubia altiora et iam nox intempesta (così stampa ora Zimmerman; diplografia di et nox);

met. 4, 31, 5 Et <statim > ipsum quod incipit velle [et statim], quasi pridem praeceperit, non moratur marinum obsequium (così stampa anche Zimmerman; diplografia di $e t$ ).

Nell'ultimo luogo in elenco la constitutio textus è di G. Ammannati $^{31}$ :

met. 2, 13, 5 at ille ubi primum consaviatus eum iuxtim se ut adsidat effecit, [attonitus et] repentinae visionis stupore <attonitus $>$ et praesentis negotii quod gerebat oblitus (così stampa Zimmerman; diplografia di et).

In base al meccanismo di riconoscimento appena esemplificato, la celebre descrizione del pappagallo indiano nei Florida potrebbe configurarsi così:

12, 1 Psittacus avis <est>Indiae [avis est]; instar illi minimo minus quam columbarum, sed color $<$ psittaco $>$ non columbarum; non enim lacteus ille vel lividus vel utrumque, subluteus aut sparsus est, sed [color psittaco] viridis et intimis plumulis et extimis palmulis, nisi quod sola cervice distinguitur. 2 Enimvero cervicula eius circulo mineo velut aurea torqui pari fulgoris circumactu cingitur et coronatur.

avis <est> Indiae [avis est] scripsi: avis Indiae avis est $\mathrm{F} \varphi$ (edd.; supplementum est attinere videtur ad avis ${ }^{1}$ : cf. iteratum avis), [avis] Indiae avis est Philomathes, avis Indiae avis est $<_{* * * *}>$ Colvius, avis Indiae [avis] est Krüger, avis Indiae talis est Rohde // color $<$ psittaco > non... [color psittaco] scripsi: colorum... color psittaco $\mathrm{F} \varphi$ (supplementum psittaco ad colorum - pro color non - attinere videtur: cf. iteratum color), $<$ nec $>$ color [um]... color psittaco Philomathes, <nec $>$ color ut... color psittaco Colvius, $<$ nec $>$ color ei... color psittaco Casaubonus, $<$ nec $>$ color ei ut... color psittaco Elmenhorstius, color non... color psittaco Hildebrand (edd.).

${ }^{31}$ La studiosa ha comunicato a Zimmerman la proposta «per litteras electronicas», come risulta dall'apparato dell'edizione oxoniense. 
Incominciamo con la strana ripetizione di avis, tollerata dall' editor princeps ma non da Philomathes, che nella Giuntina del 1522 espunge $a v i s^{1}$. Lo segue la maggior parte degli editori fino a Oudendorp, che stampa anch'egli psittacus Indiae avis est, commentando però in apparato «Sed nec male... edd. priores Psitt. avis Ind. avis est». Il giudizio dell'autorevole predecessore convince Hildebrand ${ }^{32}$ ma non Krüger, che espunge avis ${ }^{2}$, né Müller, che ripropone l'atetesi di $a v i{ }^{1}$. Un'altra via era stata imboccata da Colvius, secondo il quale dopo avis est sarebbe caduta qualche parola atta a giustificare la 'brutta' ripetizione di avis. Analoga esigenza ispira a fine Ottocento il mutamento di avis ${ }^{2}$ in talis proposto da Rohde (psittacus avis Indiae talis est), ma già ai primi del Novecento si torna con Helm alla lezione tràdita psittacus avis Indiae avis est (difesa sulla base di apol. 8, 7 una ex avibus fluvialibus amica avis ${ }^{33}$. Da allora in poi tacciono su avis... avis sia Vallette sia i successivi traduttori e commentatori, con l'unica significativa eccezione di Opeku, secondo il quale in un passo così attentamente elaborato «the repetition of avis is barely tolerable» ${ }^{34}$. A sostegno della lezione tràdita si potrebbe forse citare un passo dei Collectanea rerum memorabilium di Giulio Solino, che riecheggia, oltre a Plin. nat. 10, 117, anche il luogo dei Florida qui in discussione: 52, 43 sola India mittit avem psittacum colore viridem torque puniceo. L'indicazione in Solino sia del genere sia della specie di questo uccello pappagallo potrebbe far dubitare che già Apuleio avesse assommato in psittacus avis specie + genere, ma non persuade in ogni caso l'aggiunta di un secondo avis («L'uccello pappagallo è un uccello dell'India» $)^{35}$. Credo più probabile che avis ${ }^{2}$ sia una parola-segnale intenzionalmente ripetuta a margine da un cor-

${ }^{32}$ Che però peggiora l'interpunzione: Psittacus, avis Indiae, avis est. In apparato Hildebrand attribuisce la scelta di stampare psittacus Indiae avis est a Bosscha, e non a Oudendorp: «Oud. lectionem codd. ... non improbat, Bosscha tamen lectionem vulgatam... retinuit». Per gli interventi di Bosscha sulle annotazioni autografe di Oudendorp cfr. Magnaldi - Gianotti 2000, 2004²b, 17-18.

${ }^{33}$ Anche questo luogo, tuttavia, è dubbio (e comunque diverso da flor. 12, 1): J. Brantius ha espunto avibus, che potrebbe essere variante erronea di avis indotta dalla desinenza di fluvialibus.

${ }^{34}$ Opeku 1974, 192.

35 In Solino la parola avem è spiegabile come una sorta di lemma (Indiae aves), perché la descrizione del pappagallo si trova interposta fra quelle degli Indica maria e degli Indorum nemora. 
rettore, allo scopo di indicare che dopo avis ${ }^{1}$ andava integrato est (il copista avrebbe omesso il verbo per quasi-aplografia). La plausibilità di tale interpretazione della paradosi (avis est $=$ parola-segnale + parola da supplire) sembra suggerita dal testo che ne risulta (psittacus avis est Indiae; instar illi...), più lieve di quello edito da Helm e da Vallette e altrettanto ricco di effetti sonori.

Nello stesso passo, un'altra ripetizione o quasi-ripetizione indica forse la presenza di una seconda integrazione con parola-segnale. Si veda, dopo la pericope non enim lacteus ille vel lividus vel utrumque, subluteus aut sparsus est, con soggetto ille = psittacus, lo strano passaggio a color, soggetto sia di est sottinteso sia di distinguitur, e poi di nuovo il riferimento a psittacus di cervicula eius. I dubbi su questo faticoso andirivieni sintattico aumentano, se si confrontano con flor. 12, 1 sia il passo sopra citato di Solino sia soprattutto quello della Naturalis Historia di Plinio, fonte di entrambi: nat. 10, 117 India hanc avem mittit, siptacen vocat, viridem toto corpore, torque tantum miniato in cervice distinctam. Sia in Plinio sia in Solino, verde è l'avis e non il color $^{36}$. Un ulteriore spunto di riflessione è offerto dal precedente nonsenso colorum, sul quale si sono esercitati i migliori editori apuleiani non soltanto modificandolo in $<n e c>$ color [um] (Philomathes, seguito da Oudendorp) oppure, molto meglio, in color non (Hildebrand, «ex compendio colorñ postea in colorû commutato»), ma anche aggiungendo il dativo ei (I. Casaubonus, seguito da Elmenhorstius e Floridus). Ora, se si interpreta color $^{2}$ come una parola-segnale atta a suggerire l'integrazione dopo color(um) di psittaco, tutte le difficoltà sembrano risolte: a) al dativo illi corrisponde il dativo psittaco, due complementi di possesso: il pappagallo ha una taglia pochissimo inferiore a quella delle colombe, ma non ha lo stesso colore delle colombe; b) l'iterazione dopo psittacus di psittaco è simmetrica all'iterazione di columbarum; c) il periodo non enim... distinguitur si sviluppa saldamente e armoniosamente intorno all'unico soggetto ille: il pappagallo è tutto verde dalle piume più nascoste fino all'estremità delle ali, ma è contrassegnato da una striscia scarlatta intorno al collo (anche in Plinio distingui si riferisce all'avis e non al color).

36 Un accurato confronto tra il passo pliniano e quello apuleiano è proposto da Lee 2005, 123-125, che sottolinea l'abitudine di Apuleio a rielaborare sapientemente le sue fonti. Nel caso in discussione, tuttavia, il riferimento di viridis a color, anziché ad avis, peggiora la descrizione pliniana. 
11. Che flor. 12 sia stato trascritto da un copista stanco o distratto o alle prese con un antigrafo particolarmente impervio, ma sia stato poi scrupolosamente emendato da un correttore abituato a usare la parola-segnale, si evince anche dai successivi §§ 6-8. Anche qui il riconoscimento di un'integrazione con parola-segnale può contribuire a sanare almeno in parte il passo, uno tra i più vexati dei Florida. Per il resto ci si dovrà rassegnare alla crux, proponendo eventualmente in apparato una congettura ex ingenio, ultima di una serie molto lunga e molto variegata.

12, 6 Non enim omnibus psittacis id insigne, sed illud omnibus proprium, quod eis lingua latior quam ceteris avibus; eo facilius verba hominis articulant patentiore plectro et palato. 7 Id vero quod $<$ di $>$ dicit ita similiter nobis canit vel potius eloquitur, ut [vocem si audias] hominem putes; nam quidem <corvi vocem $>$ si audias $\dagger$ idê conate non loqui †. 8 Verum enimvero et corvus et psittacus nihil aliud quam quod didicerunt pronuntiant.

$<$ di>dicit Stewechius: dicit $\mathrm{F} \varphi^{37} / /$ vocem transposui ut supplementum attinens ad si audias ${ }^{2}$ : longe si audias Salmasius // nam quidem <corvi> scripsi: nam quidem $\mathrm{F} \varphi$, non quidem Salmasius, corvi quidem Hildebrand, $<$ corvi $>$ nam quidem van der Vliet, nam <corvum $>$ quidem Helm (Vallette), nam quidem <corvinam $>$ Coulon, nam quidem <alias $>$ Opeku // si audias F甲: si videas ed. princeps, si av<im vi>deas Oudendorp //idê conate non loqui $\mathrm{F} \varphi$ (desper. Vallette): idem conari non eloqui ed. princeps (tonare Lipsius, cantare Brantius), idem coram te eloqui Salmasius, idem conare non loqui Hildebrand, <corvum dicas $>$ idem conari non loqui Novák, idem conante $<m$ crocire $>$ non loqui van der Vliet, idem conante $<m$ corvum, conari> non loqui Purser, id est crocitare non loqui Helm (groccire in app. ex Socr. prol. 4; id est iam Fulvius), id est sonare non loqui Armini, idem conante $<s>$ non loqui Opeku, idem canente $<m>$ non loqui Capponi, idem crocitare non loqui <videtur $>$ Frassinetti, dicas crocitare non loqui Dyck $^{38}$, fortasse idem cona $<$ ntem cer $>$ te non loqui (putes subaudiri videtur).

Il passo, dedicato al pappagallo e alla sua imitazione del linguaggio umano, è accuratamente analizzato in ANRW II.34.2 da B. L. Hijmans, che elenca i numerosi emendamenti presentati per la pericope id vero... non loqui e conclude con un'affermazione scoraggiata:

${ }^{37}$ Non convince la difesa di dicit a opera di Hunink 2001, 130-131.

${ }^{38}$ Lee 2005, 35 informa che A. R. Dyck gli ha suggerito la proposta «in a personal communication». 
«[it] is not clear what point Apuleius is making» (p. 1780). In realtà, gli eccellenti filologi che si sono misurati sul testo nel corso dei secoli qualche punto fermo lo hanno stabilito. Incominciamo con la proposta di C. Salmasius ut longe si audias hominem putes: non quidem, si audias idem coram te eloqui. Essa è certo audace, come la giudica Oudendorp, ma nasce dalla giusta esigenza di rimediare in qualche modo alla strana frase tràdita vocem si audias. Perché mai Apuleio avrebbe dovuto dire del pappagallo «canta, o piuttosto pronuncia ciò che ha imparato in modo così simile a noi che, se senti la sua voce, lo crederesti un uomo», specificando un'ovvietà come «se senti la sua voce»? La diplografia di si audias ${ }^{1}$ suggerisce che si audias ${ }^{1}$ segnali l'inserimento di vocem davanti alla diplografia di si audias, e che si debba perciò scrivere ut [vocem si audias]... < <vocem $>$ si audias ${ }^{39}$.

A chi appartenga questa voce lo ha ben compreso Hildebrand. Riflettendo su verum enimvero et corvus et psittacus nihil aliud quam quod didicerunt pronuntiant, egli si chiede dapprima «qui fit, ut corvi, qui in praegressis non commemoratur, mentio fiat et uterque et psittacus et corvus in hac una re consentire dicantur, ut quae didicerint pronuntient»; e ipotizza di conseguenza che «in praegressis aliquid positum fuisse, unde diversas inter se has aves esse eluceat». Nel testo da lui stampato ut vocem si audias, hominem putes: corvi quidem si audias, idem conare, non loqui sono inaccettabili sia la terminazione attiva conare (che Hildebrand escogita «secutus vestigia codd.») sia la sostituzione di nam con corvi, ma quest'ultima parola è davvero preziosa, purché la si aggiunga a nam quidem: cfr. nam quidem <corvi vocem> si audias ${ }^{40}$. Il senso è quello già intuito da van der Vliet

39 Opeku 1974, 199 coglie la stranezza della ripetizione, ma interpreta diversamente: «My own view is that si audias has been wrongly repeated from the previous sentence, thus displacing the true reading».

${ }^{40}$ Come sopra segnalato in apparato, corvum è stampato anche da Helm (seguito da Vallette, Augello, Hunink e Lee). Poco persuasive appaiono le argomentazioni di Capponi 1987, 826-828, secondo il quale «l'ipotesi della contrapposizione della vox, educata per ammaestramento, dell'indiano psittacus, con il grido disarticolato (ved., per es., groccire) del genere corvus non ha il suffragio dell'antico empirismo», perché ne tacciono Plinio nat. 9, 117-125 e Solino collect. 52, 43-45. Anche secondo Opeku 1974, 199, non verrebbe qui nominato il corvo: «the antithesis here is more likely to be between the parrot and other talking birds than between the parrot and the raven, which is mentioned by way of example in the next sentence». 
con <corvi>nam quidem (l'aggettivo corvinam vale corvi vocem) e condiviso da $\mathrm{V}$. Coulon, che però propone nam quidem <corvinam $>$, difendendo bene nam quidem nel significato avversativo di sed sulla base di apol. 7, 7 e 33, 341. In conclusione, fra nam quidem e si audias erano probabilmente cadute due parole (corvi vocem), poi integrate a margine con diplografia dei due termini seguenti (corvi vocem si audias) e infine reimmesse in linea un po' prima del luogo di lacuna, con l'omissione più o meno intenzionale di corvi.

Per il seguito del passo, prudentemente stampato fra cruces da Vallette (e da Augello, Hunink e Lee), sembra utile tornare all'editio princeps, che stampa idem conari non eloqui (per loqui), riferendo i due infiniti al precedente putes. A conferma di conari sta il passo plautino, citato da Scioppius, Pers. prol. 9 picasque docuit nostra verba conari ${ }^{42}$. Che poi la desinenza -te del nonsenso conate nasconda non soltanto il participio presente conantem, congetturato da van der Vliet, ma anche l'avverbio certe, si può forse sospettare in base al senso e all'usus apuleiano. Si vedano i passi seguenti: flor. 16, 6 fortasse impar, certe aemulus; apol. 92, 8 vidua... iam ab alio praeflorata, certe tibi ad quae velis minime docilis; met. 2, 23, 4 hominem ferreum et insomnem, certe perspicaciorem ipso Lynceo; met. 7, 20, 1 nescio an futuris periculis me reservans, certe praesente statutaque morte liberans; Socr. 150 haud sciam an bono, certe quidem meo periculo. In tutti questi luoghi, come si vede, Apuleio impiega certe per distinguere la prima affermazione, più debole, dalla seconda, più netta e sicura. Ecco, in conclusione, la mia proposta, alleggerita dei segni di espunzione e di integrazione: Id vero quod didicit ita similiter nobis canit vel potius eloquitur, ut hominem putes; nam quidem corvi vocem si audias idem conantem, certe non loqui: «Il pappagallo canta, o piuttosto pronuncia, ciò che ha imparato in modo così simile a noi che potresti pensare sia un uomo; se invece sentissi la voce del corvo che tenta di fare lo stesso, certo non potresti pensare che parli $»^{43}$.

${ }^{41}$ Coulon 1925, 21-23.

42 In Plauto soggetto di conari sono le gazze, mentre in flor. 12, 7 non sarebbe il corvo, bensì la voce del corvo. Ma questa non sembra una difficoltà: come risulta dall'apparato, già van der Vliet e Coulon hanno proposto corvinam $(=$ corvi vocem $)$... conantem .

${ }^{43}$ Sulla possibilità che si possa sottintendere putes nella seconda parte del passo le opinioni degli studiosi moderni divergono. Opeku 1974, 199 
12. A differenza dei passi precedenti, quello che mi accingo ora a discutere non ha mai destato alcun sospetto. Tuttavia si può proporre, almeno in apparato, l'interpretazione di una dittografia quale parolasegnale atta a indicare un'antica omissione-integrazione. La proposta sembra produrre un sensibile miglioramento stilistico, come subito si vedrà (per chiarezza la inserisco direttamente nel testo).

9, 27 Prorsum enim non eo infitias nec radio nec subula nec lima nec torno nec id genus ferramentis uti nosse, sed pro his praeoptare me <reficere $>$ fateor uno chartario calamo [me reficere] poemata omnigenus apta virgae, lyrae, socco, coturno, 28 item satiras ac griphos, item historias varias rerum nec non orationes laudatas disertis nec non dialogos laudatos philosophis, atque haec et alia eiusdem modi tam graece quam latine, gemino voto, pari studio, simili stilo.

reficere transposui ut supplementum ad $m e^{1}$ attinens (cf. iteratum me).

Nel passo Apuleio antepone agli strumenti artigianali del sofista Ippia il suo unico strumento di scrittore multiforme, ovvero il calamus chartarius, la penna usata per scrivere su papiro. Grazie ad essa egli produce, rinnovandoli, componimenti di genere epico, lirico e drammatico, e poi satire, enigmi, storie, orazioni, dialoghi filosofici tanto in greco quanto in latino, con analogo spirito, pari impegno, simile stile. Hunink e Lee puntualizzano bene il particolare significato qui assunto dal verbo reficere, che non vale semplicemente «comporre», come si intendeva di solito, ma «to restore (an artefact), to repair», ovvero, come traduce Hilton, «to rework»: dunque, riprendere antichi generi letterari rielaborandoli, e riprenderli in concreto, con gli strumenti che servono a scrivere poesia (calamo e papiro) e a

si mostra dubbioso: «I suggest, therefore, nam quidem alias idem conantes non loqui, understanding putes from the earlier part of the sentence; or if this is felt to be too harsh, I would follow Novák in supplying dicas before idem». Capponi 1987, 828 ammette invece questa possibilità, proponendo nam quidem, si audias idem canentem, non loqui (ma interpretando in modo improbabile: "Apuleio avrebbe inteso dire che la vox della specie (o genere) psittacus, come sola parola articolata, potrebbe essere confusa con la voce dell'uomo, ma, come suono che ripete le stesse note o parole, non dovrebbe essere considerato humanus sermo»). Frassinetti 1991, 1207 non crede che loqui possa essere retto da putes: «Preferisco leggere, nella seconda parte, idem crocitare non loqui <videtur $>$, pensando ad un'omissione dinanzi al seguente verum». 
comunicarla (la bacchetta del rapsodo, la lira, le differenti calzature degli attori comici e tragici $)^{44}$.

Al rilievo semantico di reficere corrispondeva con ogni probabilità analogo rilievo formale. Si osservi infatti, nel segmento tràdito pro his praeoptare me fateor uno chartario calamo me reficere poemata, la ripetizione di me, sintatticamente accettabile (fateor regge l'infinitiva praeoptare me e praeoptare regge a sua volta l'infinitiva me reficere), ma piuttosto greve e per nulla necessaria (nell'unica altra occorrenza apuleiana di praeoptare con infinito il soggetto non è espresso: flor. 21, 2 adeo uti praeoptent pendere equo quam carpento sedere). Si provi ora a interpretare $m e^{2}$ come parola-segnale che suggerisce di inserire dopo $m e^{1}$ il verbo reficere $e^{45}$. Nel testo risultante pro his praeoptare me reficere fateor uno chartario calamo poemata omnigenus apta, la collocazione di reficere tra praeoptare e fateor, anziché tra calamo e poemata, sfocia in una cascata di suoni simili o identici tanto piacevoli quanto utili a spiegare la primitiva omissione di reficere per quasi-aplografia. Qui come altrove, il lepidus susurrus che accarezza le orecchie dei lettori di Apuleio sembra aver costituito un serio ostacolo per chi quelle lettere tutte uguali, susseguentisi senza posa in scriptio continua, doveva faticosamente trascriverle.

13. Un'antica integrazione con parola-segnale può forse riconoscersi anche nel passo seguente, sebbene il termine integrato, o supposto tale, si sia corrotto nella catene delle copie, tanto da rendere meno automatico il meccanismo di individuazione.

7, 5 Sed cum primis Alexandri illud praeclarum, quod imaginem suam, quo certior posteris proderetur, noluit a multis artificibus vulgo contaminari, 6 sed edixit universo orbi suo ne quis <ipse> effigiem regis temere

${ }^{44}$ Hunink 2001, 115; Lee 2005, 110, con citazione di Ulp. dig. 43, 21, 1,6 reficere est quod corruptum est in pristinum statum restaurare. Le altre occorrenze apuleiane di reficere attengono al ristoro fisico di bagno, cibo, riposo (met. 5, 8, 1; 6, 21, 3; 8, 21, 2; 10, 13, 7) o al 'rifarsi gli occhi' con uno spettacolo piacevole (met. 10, 29, 3 curiosos oculos patente porta spectaculi prospectu gratissimo reficiens). Sulla multiforme strumentazione materiale del lavoro intellettuale Apuleio si era già soffermato poco prima: 9, 24 Quin et ipse Hippian laudo, sed ingenii eius fecunditatem malo doctrinae quam supellectilis multiformi instrumento aemulari.

${ }^{45}$ L'anonimo referee preferirebbe invece considerare $m e^{2}$ quale dittografia di re(ficere). 
adsimularet aere, colore, caelamine, quin [saepe] solus eam Polycletus aere duceret, solus Apelles coloribus deliniaret, solus Pyrgoteles caelamine excuderet; 7 praeter hos tris multo nobilissimos in suis artificiis si quis uspiam reperiretur alius sanctissimae imagini regis manus admolitus, haud secus in eum quam in sacrilegum vindicaturum.

quis <ipse>... quin [saepe] scripsi: quis... quin saepe $\mathrm{F} \varphi$ (Vallette; at supplementum saepe - pro ipse sc. «per se», «ultro»- attinere videtur ad quis), quis... quin ipse Philomathes, quis... quin se[pe] Fulvius (eam deleto), quis... quin [saepe] Scioppius (Wowerius), quis...quippeni (vel quidni) saepe Stewechius, quis.. quippe saepe Heinsius, quis... quin semper Thörnell, quis...ut saepe Purser (quin $\S 6$ et ut $\S 13$ inter se permutatis), quis... [quin saepe] Müller, quis... cum saepe Leo (Helm in textu), quis... quin saepe <edixit> dub. Helm in apparatu (quis... quin saepe <scripsit> in Addendis).

Le indagini incessanti su questo passo, ispirato a Plin. nat. 7, 125 idem hic imperator edixit ne quis ipsum alius quam Apelles pingeret, quam Pyrgoteles scalperet, quam Lysippus ex aere duceret (ma con erronea sostituzione a Lisippo di Policleto, anteriore di un secolo), consentono di accantonare i dubbi sul tràdito quin, già mutato in quippeni (o in quidni) da Stewechius (e Oudendorp), in quippe da Heinsius, in $u t$ da Purser ${ }^{46}$ e in cum da Leo. Grazie a G. Thörnell, che ha raccolto luoghi paralleli «ubi quin post sententiam negativam contrarium inducens idem valet ac sed» ${ }^{47}$, si può pacificamente accogliere la difesa già svolta da Hildebrand: «Quin equidem habeo pro particula adversativa, summa cum vi asseverandi hic posita; ex praegressa vero ne particula ut saepius, cogitatione addendum $u t$, ex quo coniunctivus pendet» ${ }^{4}$.

46 Purser 1910, 147-148 ipotizza uno scambio col successivo ut di 7, 13 , dove si potrebbe scrivere quin anziché $u t . .<$ non >: «in some archetype with double columns quin and ut could have been exchanged».

47 Thörnell 1934, 151-152 cita apol. 95, 8, Socr. 138 e 155-156.

48 Così anche Hunink 2001, 97: «quin is not to be interpreted as a conjunction, but as a strongly affirmative adverb accompanying a command (duceret)». Si vedano poi le convincenti argomentazioni contro Helm, che proponeva l'aggiunta di scripsit o edixit: «If a semicolon is printed before quin, the sentence may be taken as an instance of free indirect speech (hence the subjunctive duceret), together with the following clause praeter hos... vindicaturum». Contro l'aggiunta di un verbo, e contro il mutamento di quin in cum, già si era efficacemente pronunciato Opeku 1974, 130: 
Meno convincenti risultano invece le argomentazioni di Hildebrand a favore di saepe, modificato in ipse da Philomathes (così Becichemus, Colvius, Scriverius) ed espunto da Scioppius e da Wowerius (così quasi tutti gli editori successivi, compreso Oudendorp): «Primum saepe retinui, quod primus Wowerius audacter e textu coniecit. Non unam illi artifices sed plures ut conficerent imagines Alexandrum edixisse, ea particula pronuntiatum est». Tuttavia, come obietta Opeku, «no edict could have contained a provision that certain artists were to depict him 'often'» ${ }^{49}$. Questa osservazione, del tutto condivisibile, può sfociare in una proposta diversa dall'atetesi di quin saepe, eseguita da Opeku sulla scia di Müller ${ }^{50}$, se si riflette sul testo stampato da Philomathes quin ipse solus eam Polycletus aere duceret eqs. Qui appare paleograficamente ineccepibile il ritocco di saepe in ipse, data la forte somiglianza fra le due parole, ma i criteri interni di logica e di grammatica suggeriscono di collocare ipse dopo quis, anziché dopo quin. Nel testo risultante ne quis ipse effigiem regis temere adsimularet aere, colore, caelamine, quin solus eam Polycletus aere duceret, solus Apelles coloribus deliniaret, solus Pyrgoteles caelamine excuderet, la proibizione che qualcuno ritragga Alessandro «di sua propria iniziativa» è perfettamente simmetrica all'ordine che «soltanto» Policleto, Apelle e Pirgotele ne riproducano l'immagine.

L'uso antitetico di ipse, frequente in Plauto e in Cicerone ${ }^{51}$, compare anche, per esempio, in met. 11, 23, 1 ea... partim ipse, partim per meos socios coemenda procuro. Nel passo in discussione Apuleio, fedele al proposito di reficere le opere dei predecessori $(9,27)$,

«it breaks the continuity of what appears to be a single edict from edixit universo orbi suo down to vindicaturum. Leo's cum saepe is unsatisfactory in that it removes this clause from the terms of the edict».

49 Opeku 1974, 130, con citazione di Plin. nat. 35, 93 Alexandrum et Philippum quotiens pinxerit (sc. Apelles) enumerare supervacuum est. Sorvola su quest'obiezione Hunink 2001, 97, secondo cui «Saepe does, however, add an important point: the three artists are the only ones (note the emphatically repeated solus) who are allowed to make images of the King, but they must do so frequently)». Tace sulla questione Lee.

50 Müller 1867, 463; Opeku 1974, 130: quin saepe sarebbe una glossa, spiegabile in quanto «these artists did in fact frequently portray Alexander». Altrettanto improbabile appare la modifica di saepe in semper proposta da Thörnell 1934, 151.

${ }^{51}$ Basti qui citare Plaut. Amph. 71 seu qui ipse ambissit seu per internuntium. 
potrebbe aver ripreso il pronome dalla sua fonte pliniana (edixit ne quis ipsum alius quam Apelles pingeret), riferendolo però a quis anziché ad Alessandro. Quanto al meccanismo genetico della corruttela, è possibile ipotizzare le tappe seguenti: tra quis ed effigiem un copista omette ipse per quasi-aplografia; un correttore integra a margine il pronome, con diplografia della parola precedente quis (= quis ipse); la presenza, poco dopo, di quin, simile alla parola-segnale quis, inganna chi deve eseguire in linea l'integrazione, inducendolo a trasferire dopo quin il supplemento ipse, corrotto in saepe $e^{52}$.

Giuseppina Magnaldi

Bibliografia

Ammannati = congettura di G. Ammannati citata da Zimmerman 2012.

Armini 1928 = H. Armini, Studia Apuleiana, «Eranos» 26, 1928, 273-339.

Augello 1984 = G. Augello, L'Apologia o la Magia, Florida di Lucio Apuleio, Torino 1984.

Becichemus $=$ congetture di M. Becichemus citate da Oudendorp 1823.

Brakman 1908 = C. Brakman, Apuleiana, «Mnemosyne» 36, 1908, 29-38.

Brantius = congetture di J. Brantius citate da Oudendorp 1823.

Capponi 1987 = F. Capponi, Cruces Apuleiane II, «Latomus» 46, 1987, 826-828.

Casaubonus 1594 = I. Casaubonus, L. Apuleii Apologia, Heidelbergae 1594.

Cavallo 1987 = Le strade del testo, a cura di G. Cavallo, Bari 1987.

Colvius $1588=$ P. Colvius, L. Apuleii opera omnia, Lugduni Batavorum 1588.

Coulon $1925=\mathrm{V}$. Coulon, De quelques passages altérés de l'Apologie et des Florides d'Apulée, «RPh» n. s. 49, 1925, 20-27.

De Buxis 1469 = Apuleii Madaurensis opera, cur. J. A. De Buxis, Romae 1469 (ed. princeps).

Dyck = congettura di A. R. Dyck citata da Lee 2005.

Elmenhorstius 1621 = G. Elmenhorstius, Apuleii opera omnia, Francofurti a. M. 1621.

Floridus $1688=$ J. Floridus, L. Apuleii opera in usum Delphini, Parisiis 1688.

52 Che la presenza nel contesto di una parola simile o identica alla parola-segnale abbia spesso ingannato i copisti, alle prese con un'integrazione marginale, è stato da me constatato in molte tradizioni. Si veda in ultimo Magnaldi 2012, 353-358 (su Apul. Plat. 192, 200, 236-237, 254). 
Frassinetti 1991 = P. Frassinetti, Note testuali ad Apuleio (Apol. Flor.), in Studi di filologia classica in onore di Giusto Monaco, III, Palermo 1991, 1205-1208.

Fulvius = congetture di Fulvius Ursinus citate da Oudendorp 1823.

Giarratano 1929, 1960² = C. Giarratano, Apulei Metamorphoseon libri XI, Augustae Taurinorum 1929, $1960^{2}$ cur. P. Frassinetti.

Goesius = congettura di W. Goesius citata da Oudendorp 1823.

Goldbacher 1867 = A. Goldbacher, De L. Apulei Madaurensis Floridorum quae dicuntur origine et locis quibusdam corruptis, Lipsiae 1867.

Harrison $2000=$ S. J. Harrison, Apuleius. A Latin sophist, Oxford 2000.

Harrison $2001=$ Apuleius. Rhetorical works, translated and annoted by S. J. Harrison, J. Hilton and V. Hunink, edited by S. J. Harrison, Oxford 2001.

Heinsius $=$ congetture di N. Heinsius citate da Oudendorp 1823.

Helm 1904 = R. Helm, Quaestiones Apuleianae, «Philologus» Supplementband 9, 1904.

Helm 1910, 1959² = R. Helm, Apulei Platonici Madaurensis Florida, Lipsiae $1910,1959^{2}$ cum addendis.

Hijmans 1994 = B. L. Hijmans Jr., Apuleius Orator: 'Pro se de Magia' and 'Florida', ANRW II.34.2, Berlin-New York 1994, 1708-1784.

Hildebrand 1842 = G. F. Hildebrand, L. Apuleii opera omnia, II, Lipsiae 1842 (Hildesheim 1968).

Hilton 2001 = J. Hilton, Florida, in Harrison 2001, 123-176.

Hunink 2001 = Apuleius of Madauros, Florida, edited with a commentary by V. Hunink, Amsterdam 2001.

Hunink 2001b = V. Hunink, Was Apuleius' speech stenographed? (Florida 9.13), «CQ» n. s. 51, 2001, 321-324.

Kronenberg 1908 = A. J. Kronenberg, Ad Apuleium, «CQ» 2, 1908, 304312.

Krüger $1865=$ L. Apulei Madaurensis Floridorum quae supersunt, edidit G. Krüger, Berolini 1865.

Lee 2005 = B. T. Lee, Apuleius' Florida. A Commentary, Berlin-New York 2005.

Leo $=$ congetture di F. Leo citate da Helm 1910, $1959^{2}$.

Lipsius = congetture di J. Lipsius citate da Oudendorp 1823.

Magnaldi 2000 = G. Magnaldi, La forza dei segni. Parole-spia nella tradizione manoscritta dei prosatori latini, Amsterdam 2000.

Magnaldi 2000, 2004²b = G. Magnaldi, Apologia: per una nuova collazione del Laur. 68.2 e dell'Ambros. $N 180$ sup., in Magnaldi - Gianotti 2000, 20042, 27-36.

Magnaldi 2000, $2004^{2} \mathrm{c}=\mathrm{G}$. Magnaldi, Metamorfosi: lezioni falsae ed emendatae nel Laur. 68.2, in Magnaldi, Gianotti 2000 - 2004², 37-73.

Magnaldi 2008 = G. Magnaldi, recensione di W. H. Keulen, Apuleius Madaurensis Metamorphoses Book I. Text, introduction and commentary (Groningen 2007), «ExClass» 12, 2008, 423-433. 
Magnaldi - Gianotti 2000, $2004^{2}=$ Apuleio. Storia del testo e interpretazioni, a cura di G. Magnaldi - G. F. Gianotti, Alessandria 2000, $2004^{2}$.

Magnaldi 2012 = G. Magnaldi, Tracce di antiche omissioni-integrazioni nel De Platone di Apuleio, in Vestigia notitiai. Scritti in memoria di Michelangelo Giusta, a cura di E. Bona - C. Lévy - G. Magnaldi, Alessandria 2012, 351-365.

Magnaldi - Gianotti 2000, 2004²b = G. Magnaldi - G. F. Gianotti, Codici ed edizioni, in Magnaldi - Gianotti 2000, 2004², 9-25.

Marangoni $2000=$ C. Marangoni, Il mosaico della memoria. Studi sui Florida e sulle Metamorfosi di Apuleio, Padova 2000.

Martos 2011 = J. Martos, Notes on Apuleius' Florida, «Mnemosyne» s. IV, 64, 2011, 104-109.

Müller 1867 = H. Müller, Zu Apuleius Florida, «RhM» 22, 1867, 645-648.

Nougaret $1928=\mathrm{L}$. Nougaret, Fautes et gloses dans les Florides d'Apulée, «REL» 6, 1928, 42-46.

Novák 1911 = R. Novák, Zu den philosophischen Schriften des Apuleius, «WS» 33, 1911, 101-136.

Opeku 1974 = F. Opeku, A commentary with introduction on the Florida of Apuleius, diss. London 1974.

Oudendorp 1823 = F. Oudendorp, Apuleii opera omnia, II, cur. I. Bosscha, Lugduni Batavorum 1823.

Pecere $1987=$ O. Pecere, Qualche riflessione sulla tradizione di Apuleio a Montecassino, in Cavallo 1987, 97-124 (rist. con aggiornamenti in Pecere - Stramaglia 2003, 37-60).

Pecere - Stramaglia 2003 = O. Pecere - A. Stramaglia, Studi apuleiani, Cassino 2003.

Philomathes $1522=$ L. Apuleij Madaurensis opera, cur. B. Philomathes Pisanus, Florentiae 1522 (ed. Iuntina II).

Piccioni 2011 = F. Piccioni, Un manoscritto recenziore del De magia di Apuleio: il cod. Ambrosiano N 180 Sup., «S\&T» 9, 2011, 165-210.

Purser $1907=$ L. C. Purser, Notes on the Florida of Apuleius, «Hermathena» $14,1907,360-415$.

Purser $1910=$ L. C. Purser, Notes on the Florida of Apuleius, «Hermathena» $16,1910,145-157$.

Robertson 1940-1945 = D. S. Robertson, Apulée. Les Métamorphoses, trad. P. Vallette, I-III, Paris 1940-1945 (rist. 1965-1971), II 1992², a cura di L. Callebat.

Rohde 1885 = E. Rohde, Zu Apuleius, «RhM» 40, 1885, 66-113 (rist. parziale, ma con integrazioni, in Rohde 1901, 43-74).

Rohde 1901 = E. Rohde, Kleine Schriften, II, Tübingen-Leipzig 1901 (= Hildesheim 1969).

Salmasius = congettura di C. Salmasius citata da Oudendorp 1823.

Scaliger 1600 = J. J. Scaliger, L. Apulei opera edita per Vulcanium, Lugduni Batavorum 1600.

Scioppius 1594 = G. Scioppius, Symbola critica in L. Apulei Platonici 
opera, Lugduni Batavorum 1594, rist. Augustae Vindelicorum 1605, Lugduni Batavorum 1644, Amstelodami 1664.

Scriverius 1624 = P. Scriverius, Apuleius serio castigatus, Amstelodami 1624.

Stewechius $=$ congetture di G. Stewechius citate da Oudendorp 1823.

Thörnell 1934 = G. Thörnell, Analecta critica, «Eranos» 32, 1934, 142158.

Tollius = congettura di J. Tollius citata da Oudendorp 1823.

Toschi $2000=$ Apuleio neosofista. Discorso per la sua statua a Cartagine (Floridorum 16), introduzione, testo, traduzione e commento a cura di A. Toschi, Parma 2000.

Vallette 1924, $1960^{2}=$ Apulée. Apologie. Florides, texte établi et traduit par P. Vallette, Paris 1924, $1960^{2}$.

van der Vliet $1900=$ L. Apuleii Madaurensis Apologia sive de magia liber et Florida, recensuit J. van der Vliet, Lipsiae 1900.

Vössing 2008 = K. Vössing, Exceptum instead of excerptum in Apuleius, Florida 9.13, «CQ» n. s. 58, 2008, 395-397.

Vossius $=$ congettura di G. Vossius citata da Oudendorp 1823.

Vulcanius 1594 = B. Vulcanius, L. Apulei opera omnia, Lugduni Batavorum 1594.

Wowerius 1606 = J. Wowerius, L. Apulei opera, Hamburgi 1606.

Zimmerman 2012 = Apulei Metamorphoseon libri XI, recognovit brevique adnotatione critica instruxit M. Zimmerman, Oxonii 2012. 